PROCEEDINGS OF THE

AMERICAN MATHEMATICAL SOCIETY

Volume 46, Number 1, October 1974

\title{
A NOTE ON METRIC-FINE SPACES
}

\section{ZDENĚK FROLÍK}

ABSTRACT. The coreflection into metric-fine spaces $X$ is explicitly described, and it is shown that metric-fine proximally fine spaces are just the spaces $X$ such that $f: X \rightarrow Y$ is uniformly continuous whenever the pre-images under $f$ of zero sets are zero sets.

Basic properties of metric-fine and (separable metric)-fine spaces are derived, and the corresponding coreflections are described using apparently a new concept of a complete projective family with respect to a class of spaces. In conclusion the uniform spaces determined by cozero sets are identified as metric-fine proximally fine spaces.

The notion of metric-fine spaces (introduced by A. Hager [1]) seems to be very useful in the theory of uniform spaces. If we add to metric-fine some property we usually get a very interesting notion. One example is given in this note. More examples are given in subsequent papers; e.g., measurable uniform spaces are just hereditarily metric-fine spaces (see Frolik [3]).

Let $F$ be a coreflection of a category $\mathcal{Q}$, and let $\mathcal{K}$ be a class of objects of the category $\mathscr{A}$. A $\mathcal{K}-F$ object is an object $A$ such that any morphism of $A$ into an object $K$ in $\mathcal{K}$ factorizes (uniquely) through the coreflection $F K \rightarrow$ $K$. By a theorem of J. Vilímovský [1], the class of all $\mathcal{K}-F$ objects is coreflective provided that $\mathfrak{Q}$ fulfils the natural conditions.

This note concerns the category of uniform spaces. In general we use the terminology and notation of E. Cech [1].

Following Isbell [1], denote by $\alpha$ the usual fine coreflection, and denote by $e$ the usual reflection onto separable uniform space. Thus $\alpha X$ is the underlying set $X$ of $X$ endowed with the (uniformly finest) uniformity topologically equivalent to $X$, and $e X$ is $X$ endowed with the uniformity which has all countable uniform covers of $X$ for a bas is of uniform covers.

Received by the editors March 2, 1973.

AMS (MOS) subject classifications (1970). Primary 54E10, 54E15.

Key words and phrases. Fine, proximally fine, metric-fine and coz-fine uniformities, completely additive, cozero covers, $\sigma$-uniformly discrete, coreflection, metrically determined functors. 
In accordance with the general definition, a uniform space will be called metric $f$ ine (or (separable metric)-fine) if for every uniformly continuous mapping $f$ of $X$ into a metric (or separable metric, resp.) space $M$ the mapping $f: X \rightarrow a M$ is uniformly continuous. By Vilimovský [1] the categories of metric-fine and (separable metric)-fine spaces are coreflective.

In this note the coreflections are explicitly described, and as a byproduct the coreflectivity is obtained. The following lemma will be used just for $\beta=\alpha$.

Definition. Let $A$ be an object of a category $\mathcal{Q}$, and let $\mathcal{K}$ be a class of spaces. A projective family $\left\{f_{a}: A \rightarrow A_{a}\right\}$ is called $\mathcal{K}_{\text {-complete }}$ if for every morphism $f: A \rightarrow K, K \in \mathcal{K}$, there exists an $a$, and a $g: A_{a} \rightarrow K$ such that $f=g \circ f_{a}$.

Lemma. Assume that $\mathcal{K}$ is a class of metric spaces which is closed under countable products and subspaces, and let $\beta$ be a coreflection of uniform spaces such that $\beta M \rightarrow M$ are identities.

Let $X$ be a uniform space, and let $Y$ be projectively generated by all mappings $f: Y \rightarrow \beta M$ where $f: X \rightarrow M$ is uniformly continuous, $M \in \mathcal{K}$. Then the family $\{f: Y \rightarrow \beta M\}$ is K-complete.

Proof. If a space $Y$ is projectively generated by a family of mappings $\left\{f_{a}: Y \rightarrow Y_{a}\right\}$, then, by definition, the pre-images of uniform covers of $Y_{a}$ 's under $f_{a}$ 's form a subbasis for the uniform covers of $Y$. Then each uniform cover of $Y$ is realized (is refined by the pre-image of a uniform cover) by the reduced product $f$ of a finite family $\left\{f_{a} \mid a \in A\right\}, f: Y \rightarrow \Pi\left\{Y_{a} \mid a \in A\right\}$, and hence each countable collection of uniform covers of $Y$ is realized by such an $f$ with $A$ countable.

Now let $h$ be a uniformly continuous mapping of $Y$ onto an $S \in \mathcal{K}$. Take an $f$ as above such that the pre-image (under $h$ ) of each $1 / n$-cover is realized by $f$. We have $Y_{a}=\beta M_{a}, M_{a} \in \mathcal{K}$, and $f_{a}: X \rightarrow M_{a}$ are uniformly continuous. Let $M$ be the subspace $f[X]$ of the product of $\left\{M_{a}\right\}$. Then $f: X \rightarrow M$ is uniformly continuous, and $f: Y \rightarrow \beta M$ is one of the generating maps for $Y$.

Since $\beta$ is a coreflection, the identity mapping of $\beta M$ in the product of $\left\{\beta M_{a}\right\}$ is uniformly continuous, and hence $f: Y \rightarrow \beta M$ realizes the pre-image of each uniform cover of $S$ under $h$, and hence there is a uniformly continuous mapping $g: \beta M \rightarrow S$ such that $h=g \circ f$. This concludes the proof.

If $X$ is a uniform space we denote by $\operatorname{coz} X$ the collection consisting of the cozero sets of uniformly continuous real valued functions on $X$. Obviously, $\operatorname{coz} X$ is closed under the operations of taking finite intersections and 
countable unions. If $f: X \rightarrow Y$ is uniformly continuous then the pre-images of cozero sets are cozero sets.

The complements of cozero sets are the zero sets; and the collection of all zero sets is denoted by $z X$.

For the proof of Theorem 1 we need the following simple observation:

If $X$ is a uniform space then completely $\operatorname{coz} X$-additive uniform covers form a bas is for the uniform covers of $X$.

Theorem 1. For each uniform space $X$ let $m_{\boldsymbol{N}_{0}} X$ be the uniform space projectively generated by the identity mapping onto $X$, and by all $f: m_{\boldsymbol{N}_{0}} X \rightarrow$ $\alpha M$ where f runs over all uniformly continuous mappings into separable metric spaces M. Then:

(1) The uniform space $Y$ projectively generated by all the $f: Y \rightarrow x M$ has for a basis of uniform covers the countable covers by cozero sets in $X$.

(2) $m_{\aleph_{0}} X$ has for a basis of uniform covers the covers of the form $\left\{V_{a} \cap B_{n} \mid n \in N, a \in A\right\}$ where $\left\{B_{n}\right\}$ ranges in $\operatorname{coz} X$, and $\left\{V_{a}\right\}$ is a uniform cover of $X$ (which may be assumed to be completely coz $X$-additive).

(3) $Y=e m_{\kappa_{0}} X=m_{\boldsymbol{\kappa}_{0}} e X$.

(4) If $X$ is separable then $m_{\boldsymbol{\kappa}_{0}} X$ has countable covers ranging in coz $X$ for the basis of uniform covers.

(5) The family $\left\{f: m_{\boldsymbol{x}_{0}} X \rightarrow a M\right\}$ with $f$ being uniformly continuous mappings of $X$ onto separable metrizable spaces, is separable metric-complete.

(6) $m_{\boldsymbol{\kappa}_{0}} m_{\boldsymbol{\kappa}_{0}} X=m_{\boldsymbol{\kappa}_{0}} X$.

(7) $m_{\kappa_{0}} X$ is a coreflection of $X$ in (separable metric)-fine spaces.

Theorem 2. The following three properties of a space $X$ are equivalent:

(1) $X$ is (separable metric)-fine.

(2) If $f$ is a mapping of $X$ into a separable metric space $Y$ such that the pre-images of cozero sets are cozero sets, then $f$ is uniformly continuous.

(3) Condition (2) with "metric" deleted.

Proof of Theorem 1. (1) We may and shall assume that $e X=X$. Then we may apply Lemma 1 to obtain that every uniform cover of $Y$ is realized by one of the mappings $f: Y \rightarrow a M$ where $M$ is a separable metrizable space. Hence the pre-images of open countable covers of $M$ 's form a basis for uniform covers of $Y$. Since the pre-images of the open sets in $M$ 's are cozero sets in $X$, it remains to show that every countable cover ranging in $\operatorname{coz} X$ is uniform (in $Y$ ). Let $\left\{B_{n}\right\}$ be any countable cover ranging in $\operatorname{coz} X$. Choose 
uniformly continuous functions $f_{n}$ such that $B_{n}$ is the cozero set $\left\{x \mid f_{n} x \neq 0\right\}$. Let $f$ be the reduced product of $\left\{f_{n}\right\}$, and let $M=f[X] \subset R^{N}$. The map $f: X \rightarrow M$ is uniformly continuous, $B_{n}=f^{-1}\left[f\left[B_{n}\right]\right]$, and $f\left[B_{n}\right]$ is an open cover of $M$, hence a uniform cover of $\alpha M$. This concludes the proof of (1).

(2) By (1) the covers described in (2) form a subbasis for the uniform covers of $m_{\mathrm{N}_{0}} X$. It is routine to show that any two of them have a common star-refinement, and hence the covers form a basis.

(3) It follows directly from the definition that $Y=m_{\kappa_{0}} e X$, and that $e m_{\mathrm{N}_{0}} X$ is finer than $Y$. It remains to show that $Y$ is finer than $e m_{\kappa_{0}} X$. Thus we must show that any countable uniform cover $\left\{W_{k}\right\}$ of $m_{x_{0}} X$ is refined by a countable cover $\left\{C_{m}\right\}$ ranging in $\operatorname{coz} X$. By (2) $\left\{W_{k}\right\}$ is refined by a cover described in (2). Put

$$
\begin{aligned}
C_{n k}= & B_{n} \cap \bigcup\left\{V_{a} \mid V_{a} \cap B_{n} \subset W_{k}\right\} \\
& =B_{n} \cap \bigcup\left\{V_{a} \cap B_{n} \mid V_{a} \cap B_{n} \subset W_{k}\right\} .
\end{aligned}
$$

Clearly $\left\{C_{n k}\right\}$ is a countable cover of $X$, and it ranges in $\operatorname{coz} X$ if $\left\{V_{a}\right\}$ is completely coz $X$-additive. This concludes the proof.

(4) is a consequence of (2).

(5) follows from (1) and (3).

(6) follows from (5).

(7) It follows from (6) that $m_{\boldsymbol{N}_{0}} X$ is a (separable metric)-fine space. Let $f$ be a uniformly continuous mapping of a (separable metric)-fine space $Z$ into $X$. We must show that $g: Z \rightarrow m_{\mathrm{N}_{0}} X$ is uniformly continuous. So it is enough to show that $g: Z \rightarrow Y$ followed by each of the generating mappings for $Y=m_{\kappa_{0}} X$ is uniformly continuous. This is obvious for the identity mapping of $Y$ onto $X$, and if $f: Y \rightarrow a M$ is any other generating mapping then $g f: X \rightarrow M$ is uniformly continuous, hence $f \circ g: Z \rightarrow M$ is uniformly continuous, hence $f \circ g: Z \rightarrow a M$ is uniformly continuous because $Z$ is (separable metric)-fine. This concludes the proof.

Proof of Theorem 2. Conditions (1) and (2) are equivalent by Theorem 1 . Obviously (3) implies (2). Every separable uniform space is projectively generated by mappings into separable metric spaces, and hence (2) implies (3).

In the next theorem the basic facts about metric-fine spaces are given. We use the theorem of $\mathrm{A} . \mathrm{H}$. Stone [1] which says that every open cover of a metric space has a $\sigma$-uniformly discrete open refinement. 
Theorem 3. For each uniform space $X$ let $m X$ be the uniform space projectively generated by the family of all mappings $f: m X \rightarrow$ aM where $f$ is a uniformly continuous mapping of $X$ onto a metric space $M$. Then

(1) $\{f: m X \rightarrow \alpha M\}$ is a metric-complete family.

(2) $m X$ is a coreflection of $X$ in the category of metric-fine spaces.

(3) $m X$ has for a basis of uniform covers the covers of the form $\left\{B_{n} \cap U_{a}^{n} \mid\right.$ $\left.n \in N, a \in A_{n}\right\}$ where $\left\{U_{q}^{n} \mid a \in A\right\}$ are uniform covers of $X$ and $\left\{B_{n}\right\}$ ranges in coz $X$. In addition we may assume that the cover is completely coz $X$ additive.

(4) $m X$ has for a bas is of uniform covers the covers in (3) with the additional property: $\left\{B_{n} \cap U_{a}^{n} \mid a \in A\right\}$ is uniformly discrete in $X$.

(5) $\operatorname{coz} X=\operatorname{coz} m_{\boldsymbol{\kappa}_{0}} X=\operatorname{coz} m X$.

Theorem 4. For any space $X: e m X=e m_{\boldsymbol{N}_{0}} X=m_{\boldsymbol{\kappa}_{0}} e X$.

Proof. Clearly $e m X$ is finer than $e m_{\boldsymbol{K}_{0}} X$, and the converse follows from property (5) in Theorem 3, and description (4) (in view of (3)) in Theorem 1 of $e m_{\boldsymbol{N}_{0}} X$. Thus $e m X=e m_{\boldsymbol{\kappa}_{0}} X$. From the description of the covers in Theorems 1 and 3 it follows that $m e X=m_{\boldsymbol{\kappa}_{0}} e X$. By Theorem $1, e m_{\boldsymbol{\kappa}_{0}} X=m_{\boldsymbol{\kappa}_{0}} e X$.

Proof of Theorem 3. Condition 1 follows from the Lemma, clearly (1) implies $m m X=m X$, and (3) follows from this and (1) by an argument similar to that in the proof of Theorem 1. Namely, one shows that $m X$ is metricfine, and if $g: Z \rightarrow X$ is uniformly continuous and $Z$ is metric-fine, then $g: Z \rightarrow m X$ followed by each generating $f: m X \rightarrow \alpha M$ is uniformly continuous, hence $g: Z \rightarrow m X$ is uniformly continuous.

One gets the covers in (3) and (4) as the pre-images of covers from M's under generating $f: m X \rightarrow \alpha M$ 's as follows:

If $\mathcal{U}$ is any open cover of $M$, then by $A . H$. Stone's theorem there exists a $\sigma$-uniformly discrete open refinement $\left\{V_{a}^{n} \mid a \in A_{n}, n \in N\right\}$ of $\mathcal{U}$. Put

$$
\begin{aligned}
& C_{n}=\bigcup\left\{V_{a}^{n} \mid a \in A_{n}\right\}, \quad U_{a}^{n}=f^{-1}\left[V_{a}^{n}\right], \\
& B_{n}=f^{-1}\left[C_{n}\right]=\bigcup\left\{U_{a}^{n} \mid a \in A_{n}\right\} .
\end{aligned}
$$

Since $\left\{V_{a}^{n} \mid a \in A_{n}\right\}$ is uniformly discrete in $M$, say with $r>0$, the trace of the r-cover on $C_{n}$ refines $\left\{V_{a}^{n}\right\}$, and hence $\left\{V_{a}^{n} \mid a \in A\right\}$ is a trace of $C_{n}$ of a uniform cover of $M$. Thus $\left\{U_{a}^{n}\right\}$ is a trace on $B_{n}$ of a uniform cover of $X$ (the map $f: X \rightarrow M$ is uniformly continuous). Thus $\left\{U_{a}^{n} \cap B_{n}\right\}$ has required properties and refines $f^{-1}[\mathcal{U}]$.

It remains to show that every cover in (3) is a uniform cover of $m X$. 
Realize all covers $\left\{U_{a}^{n}\right\}$ and all $B_{n}$ by a uniformly continuous mapping $f: X \rightarrow$ $M, M$ metric. Then the cover in (3) is refined by the pre-images of an open cover of $M$.

To prove property (5) we shall show that every countable uniform cover $\left\{W_{k}\right\}$ of $m X$ is refined by a countable cover $\left\{C_{m}\right\}$ ranging in $\operatorname{coz} X$. We know that $\left\{W_{k}\right\}$ is refined by a cover in statement (3) in Theorem 3. Put

$$
C_{k n}=\bigcup\left\{U_{a}^{n} \cap B_{n} \mid U_{a}^{n} \cap B_{n} \subset W_{k}\right\}
$$

It is easily seen that the cover $\left\{C_{k n}\right\}$ ranges in $\operatorname{coz} X$ (because $\left\{U_{a}^{n}\right\}$ are completely coz-additive), and refines $\left\{W_{k}\right\}$.

Thus $e m X=e m_{\boldsymbol{\kappa}_{0}} X$ by Theorem 1, and hence $\operatorname{coz} m X=\operatorname{coz} e m X=$ $\operatorname{coz} e m_{\kappa_{0}} X=\operatorname{coz} X$ by application of Theorem 1 .

Corollary. A space $X$ is metric-fine if and only if each $\sigma$-uniformly discrete completely coz-additive cover is uniform. For any space $X$ the $\sigma$ uniformly discrete completely coz-additive covers form a basis for the uniform covers of $m X$.

Proof. The covers in Theorem 3, condition (4), are just $\sigma$-uniformly discrete completely coz-additive covers.

A coz-morphism of a uniform space $X$ into a uniform space $Y$ is a mapping $f: X \rightarrow Y$ such that the pre-images of cozero sets in $Y$ are cozero sets in $X$. Clearly every uniformly continuous mapping is a coz-morphis $m$, and the converse is not true. By The orem 2 every coz-morphism of a metric-fine space into a separable uniform space is uniformly continuous. We shall describe all spaces $X$ for which any coz-morphism $f: X \rightarrow Y$ is uniformly continuous.

Recall that a uniform space $X$ is called proximally fine if the uniformity is the finest one (uniformly) compatible $w$ ith the induced proximity. Denote by $p X$ the precompact uniform space associated with $X$. Clearly $p$ is a reflection, and the finite uniform covers of $X$ form a basis for uniform covers of $p X$.

One can show immediately that a uniform space is proximally fine if and only if for every uniformly continuous mapping $f$ of $X$ into $p Y$ where $Y$ is metrizable, $f: X \rightarrow Y$ is uniformly continuous.

By an (A.D.) Alexandrov space we mean a uniform space $X$ such that every bounded uniformly continuous coz-morphism into the real line is uniformly continuous. We note that $X$ is an Alexandrov space if and only if every finite cozero cover of $X$ is uniform. 
Theorem 5. The following five properties of a uniform space $X$ are equivalent:

1. If $f$ is a coz-morphism of $X$ into a uniform space $Y$ then $f: X \rightarrow Y$ is uniformly continuous.

2. Condition 1 with Y metrizable.

3. $X$ is metric-fine and proximally fine.

4. $X$ is (separable metric)-fine and proximally fine.

5. $X$ is a proximally fine Alexandrov space.

Proof. Obviously (1) implies (2), and (3) implies (4). By Theorem 2, condition (4) implies (5). We shall show that (2) implies (3), and (5) implies (1).

First we need a simple observation. Let $f$ be a mapping of $X$ into a metric space $Y$. Then it is easily seen that if one of the mappings $f: X \rightarrow$ $Y, f: X \rightarrow p Y, f: X \rightarrow a Y$ is a coz-morphism, then all three mappings are coz-morphisms.

Assume condition (2), and let $f$ be a mapping of $X$ into a metrizable uniform space $Y$. If $f: X \rightarrow Y$ is uniformly continuous, then $f: X \rightarrow Y$ is a coz-morphism, hence $f: X \rightarrow \alpha Y$ is a coz-morphism, and by condition (2) the mapping $f: X \rightarrow a Y$ is uniformly continuous. Hence $X$ is metric-fine. Now let $f: X \rightarrow p Y$ be uniformly continuous. Hence $f: X \rightarrow p Y$ is a cozmorphism, hence $f: X \rightarrow Y$ is a coz-morphism, and by condition (2), uniformly continuous. This proves that $X$ is proximally fine.

Assume condition (5), and let $f$ be a coz-morphism of $X$ into a space $Y$. Since $X$ is an Alexandrov space, $f: X \rightarrow p Y$ is uniformly continuous by definition because $p Y$ is projectively generated by bounded functions, and since $X$ is proximally fine, $f: X \rightarrow Y$ is uniformly continuous. This concludes the proof.

Definition. A uniform space $X$ is said to be coz-fine if it satisfies the equivalent conditions in The orem 5 .

Theorem 6. A uniform space $X$ is coz-fine if and only if for every mapping $f$ of $X$ into a metrizable uniform space $Y$, if $f: X \rightarrow p Y$ is uniformly continuous then $f: X \rightarrow a Y$ is uniformly continuous.

Proof. Theorem 5.

Theorem 7. The category of coz-fine spaces is coreflective.

Proof. It is enough to show that if a space $X$ is inductively generated by a family of mappings $f_{a}: X_{a} \rightarrow X$ with $X_{a}$ 's coz-fine then $X$ is coz-fine. 
Let $f: X \rightarrow p Y$ be uniformly continuous with $Y$ metrizable. Hence all $f \circ f_{a}: X_{a} \rightarrow p Y$ are uniformly continuous, by Theorem 6 all the mappings $f \circ f_{a}: X_{a} \rightarrow a Y$ are uniformly continuous, and since $\left\{f_{a}\right\}$ is an inductively generating family, $f: X \rightarrow \alpha Y$ is uniformly continuous.

Remark. It is obvious that if $F$ and $G$ are two functors on uniform spaces, which preserve the underlying set, and if $\mathcal{K}$ is a class of spaces, and if $\mathbb{P}$ is the class of all spaces $X$ such that uniform continuity of $f: X \rightarrow$ $F K, K \in \mathcal{K}$, implies the uniform continuity of $f: X \rightarrow G K$, then $\mathscr{Q}$ is coreflective. Certainly, one can get more general results from the proof of Theorem 7.

An explicit description of the coreflection will be given in a subsequent paper on locally fine uniform spaces. We need the results of this note in a paper on measurable spaces.

In conclusion we show by a simple example that a coz-fine space need not be fine.

Let $D$ be an uncountable set, and let $\mathcal{B}$ be the $\sigma$-algebra on $D$ which consists of all countable subsets of $D$ and their complements. Let $X$ be the set $D$ endowed with the uniformity having all completely $B$-additive partitions (such partitions must be countable!) for a basis of uniform covers. It is obvious that $X$ is topologically discrete. On the other hand we shall show that if $f$ is a coz-morphism of $X$ onto a metric space $Y$, then $\left\{f^{-1}[(y)] \mid y \in Y\right\}$ is a uniform cover of $X$, and hence $f: X \rightarrow Z$ is uniformly continuous when $Z$ is the set $Y$ given the finest uniformity. Hence $X$ is coz-fine.

First observe that $Y$ is a separable metric space: if $\left\{U_{a}\right\}$ is a disjoint family of open sets in $Y$, then $\left\{f^{-1}\left[U_{a}\right]\right\}$ is a completely $\Re$-additive disjoint family in $X$, and hence it is countable.

Let $U$ be the union of all open sets $V$ in $Y$ such that $f^{-1}[\mathrm{~V}]$ is countable. Since $U$ is a cozero set, the set $X=f^{-1}[U]$ must be countable. The complement of $U$ is nonvoid, and it contains at most one point. Hence $Y-U$ is a singleton $(z)$. Hence $\left\{f^{-1}(y) \mid y \in Y\right\}$ is a uniform cover of $X$.

Remark. The Borel sets on the real line, in general, Baire sets in any analytic topological space (in particular, any compact space) gives coz-fine uniformity. One must know that completely additive partitions are countable (and this is not easy). This follows from the main result in Frolik [4].

Theorem 5 is a refinement of results in Frolik [1]. The results of this note were included in the author's lecture at the Second International Topological Symposium on Topology in Budva, August 1972.

In conclusion it is my pleasant duty to note that M. Rice obtained the main part of Theorem 3 in his dissertation (Wesleyan University, 1973), and A. Hager found a part of Theorem 5 . 


\section{REFERENCES}

E. Čech

1. Topological spaces, 2nd ed., Publ. House Czech. Acad. Sci., Prague, 1966; English transl., Wiley, New York, 1966. MR 35 \#2254.

Z. Frolík

1. Topological methods in measure theory and measurable spaces (Proc. Third Prague Sympos., 1971), Academia, Prague, 1972.

2. Interplay of measurable and uniform spaces, Proc. Second Internat. Sympos. on Topology and Appl., Budva, 1972.

3. Measurable uniform spaces, Pacific J. Math. (to appear).

4. A measurable map with analytic domain and metrizable range in quotient, Bull. Amer. Math. Soc. 76 (1970), 1112-1117. MR 42 \#448.

A. Hager

1. Some nearly fine uniform spaces, Proc. London Math. Soc. (to appear).

2. Measurable uniform spaces, Fund. Math. 77 (1972), 51-73.

J. Isbell

1. Uniform spaces, Math. Surveys, no. 12, Amer. Math. Soc., Providence, R. I., 1964. MR $30 \# 561$.

DEPARTMENT OF MATHEMATICS, UNIVERSITY OF PITTSBURGH, PITTSBURGH, PENNSYLVANIA 15213

MUKU, SOKOLOVSKA 83, PRAGUE, CZECHOSLOVAKIA 